\title{
Novel Mechanisms for Resveratrol and Metformin: Implications in Alzheimer's Disease
}

\author{
M. Guo ${ }^{1}$, A. Calabro ${ }^{2}$, C. Queenan ${ }^{2}$, D. Leonardi ${ }^{1}$ \\ 1. Bergen County Academies, Cell Biology Lab, 200 Hackensack Avenue, Hackensack, NJ 07601 \\ 2. Bergen County Academies, Nano-Structural Imaging Lab, 200 Hackensack Avenue, Hackensack, NJ \\ 07601
}

The leading hypothesis for the cause of Alzheimer's disease (AD) is chronically high levels of synapsedisrupting $\beta$-amyloid $(A \beta)$ peptide fragments in the brain. Recently, the relationship between AD and diabetes has been the focus of extensive investigation. Several studies have shown that the risk of developing $\mathrm{AD}$ is increased 50-100\% in patients with type 2 diabetes mellitus (T2D) [1]. The insulin sensitizing drug, metformin (Glucophage ${ }^{\mathrm{R}}$ ), was initially hypothesized to reduce $\mathrm{A} \beta$ generation and accumulation, but has since been shown to significantly increase the generation of both intracellular and extracellular $A \beta$ species in vitro [2]. Using neuroblastoma cells (N2a-695) stably expressing human amyloid precursor protein (APP), some research has shown that metformin causes an increase in $A \beta$ through the upregulation of BACE1 transcription [2].

Alternative treatment strategies and/or molecular targets to reverse the effects of $\mathrm{A} \beta$ are warranted. Resveratrol, a polyphenol found in the skin of red grapes, has been reported to promote autophagy, a process in which the cell lysosomally degrades dysfunctional cellular components and employs these products as building blocks or energy sources [3]. Recently, resveratrol has been reported to function by stimulating AMPK and cause the degradation of $A \beta$ in vitro by autophagic processing [4]. The reactive oxygen species, superoxide, has been shown to disrupt the normal processing of APP [5]. The antioxidant properties of resveratrol may alleviate the disruption of APP. These findings suggest the therapeutic value of resveratrol in the treatment of AD potentially exacerbated by metformin intake.

The objectives of this study were to perform viability assays to determine concentrations of resveratrol and metformin treatments that would not exhibit significant toxicity to cells, perform an A $\beta 42$ ELISA to quantitatively measure $A \beta$, assess the mechanism by which metformin and resveratrol affect $A \beta$, and observe cell morphology. Data were corrected to \% of control per cell using viability assays.

N2a-695 mouse neuroblastoma cells were transfected to produce human APP. Measurement of p62 was used to quantify autophagy and activation of AMPK; transmission electron microscopy was used for qualitative analysis (Fig. 1). The data suggested that metformin $(20 \mathrm{mM})$ causes aberrant autophagy, which leads to $\beta$-amyloid accumulation $(\mathrm{p}<0.01)$. Resveratrol $(5 \mu \mathrm{M})$ significantly reduced $\beta$-amyloid in cells treated with metformin, however via an AMPK-independent pathway $(p<0.01)$ with no effect on autophagy in this model system. Measurement of mitochondrial superoxide by fluorescence microscopy indicated that resveratrol causes a decrease in superoxide (Fig. 2), as shown by a decrease in fluorescent signal, suggesting a contribution of mitochondrial integrity in the ability of cells to degrade $A \beta(p<0.01)$. Furthermore, the combination treatment of resveratrol and metformin caused the decrease of caspases $3 / 7$ suggesting that the combination of the two compounds has neuroprotective properties $(\mathrm{p}<0.01)$. This research identifies an AMPK-independent pathway through which resveratrol reduces $\beta$-amyloid levels which may have potential as a viable treatment option for AD. 
References:

[1] G. Biessels, et al., The Lancet Neurology 1 (2006) 64-75.

[2] T. Chen, et al., PNAS 106 (2009) 3907-3912.

[3] N. Gurusamy, et al., Cardiovascular Research 86 (2009) 103-112.

[4] V. Vingtdeux, et al., The Journal of Biological Chemistry 125 (2010) 2115-2125.

[5] C. Massaad, et al., Aging 1 (2009) 758-761.

[6] The authors would like to acknowledge the administration of the Bergen County Technical Schools for their continued support of the research program.
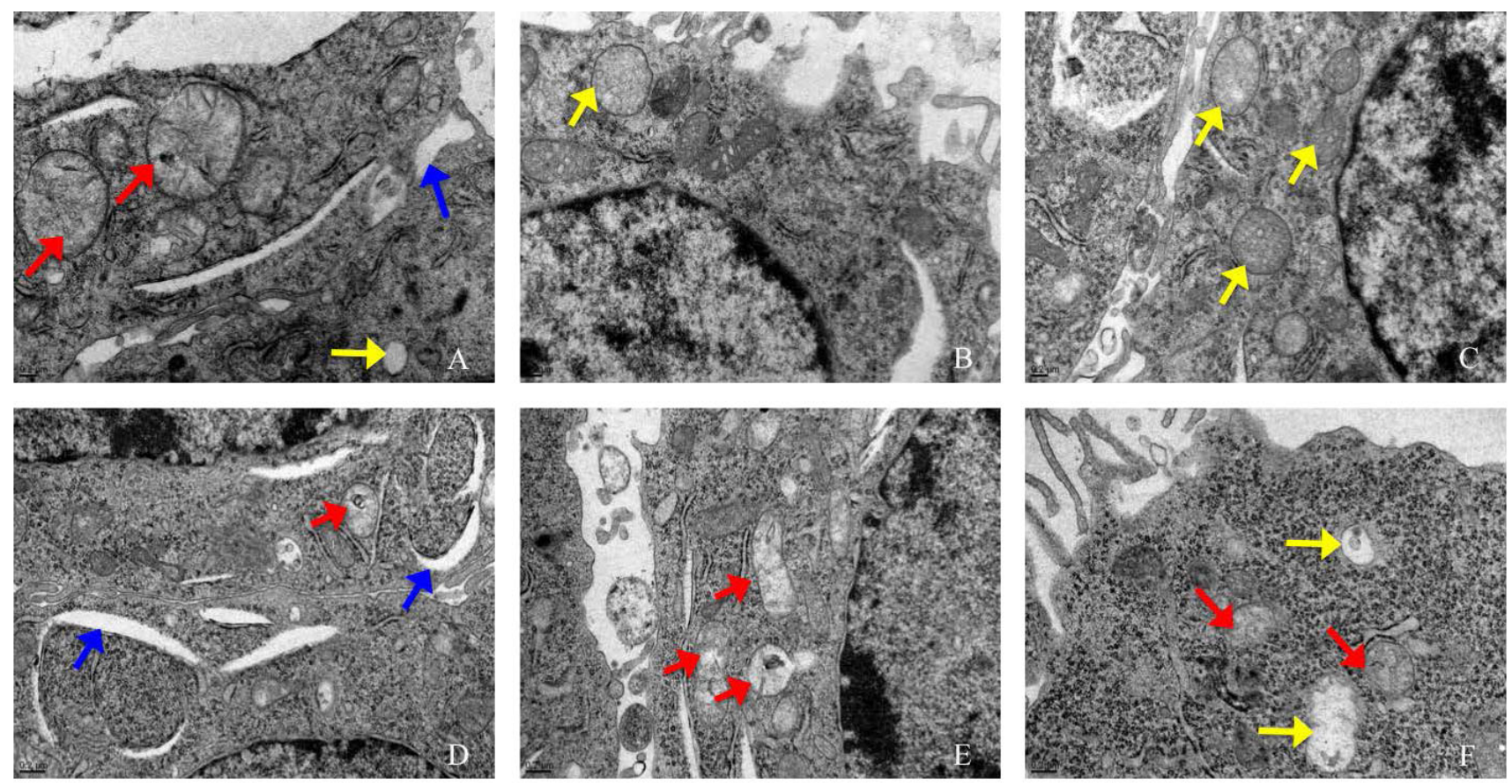

Figure 1. Transmission electron microscopy of N2a-695 cells. Untreated cells (A, B, C) and cells treated with $5 \mu \mathrm{M}$ resveratrol for 24 hours (D, E, F). Red arrows indicate mitochondria, yellow arrows indicate lysosomes, and blue arrows indicate autophagosome formation. Scale (lower left) $=0.2 \mu \mathrm{m}$.
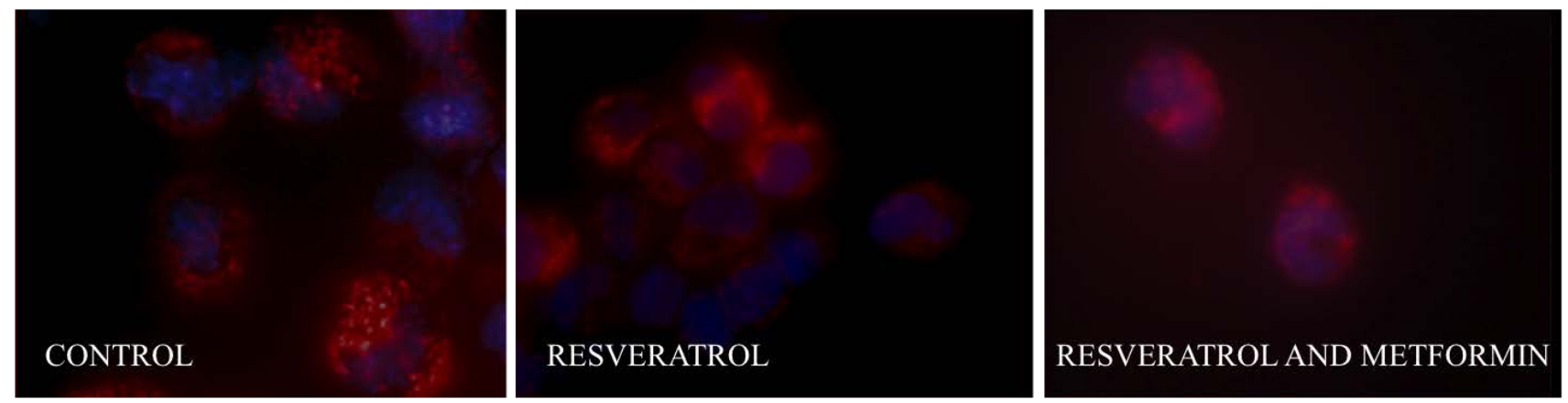

Figure 2. Fluorescence microscopy of N2a-695 cells to observe superoxide production. Cells were stained to observe mitochondrial superoxide production (red fluorescence) and Hoechst nuclear stain (blue fluorescence). Control image 630X magnification; resveratrol, and resveratrol and metformin images taken at 1000X magnification using a Nikon Eclipse 80i microscope. 